\title{
Editorial
}

\section{Anti-NMDA receptor encephalitis: clinical and research implications}

Anti-NMDA receptor encephalitis is a severe neuropsychiatric disease, caused by autoimmune antibodies against $\mathrm{N}$-methyl-D-aspartate glutamate receptors (NMDAR). Although clinical descriptions suggest that anti-NMDAR encephalitis has existed for centuries, this disease was formally recognised following the publication of a case series in 2007 (1). Clinical presentation of anti-NMDAR encephalitis may vary widely, but it often includes the following four phases, prodromal phase, psychotic and/or seizure phase, unresponsive phase and hyperkinetic phase (2). These clinical phases may manifest with a plethora of psychiatric presentations encompassing delirium, psychosis, catatonia, affective syndromes and anxiety. Patients with anti-NMDAR encephalitis may initially arrive at the emergency psychiatric services or at the psychiatric intensive care units with only psychiatric symptoms and may pose major diagnostic challenges to the treating psychiatrists (3).

Anti-NMDAR encephalitis is not rare (2). Prevalence of anti-NMDAR antibodies among the first episode patients, who met the DSM-IV diagnostic criteria for schizophrenia, has been reported as $6.5 \%$ (4). Anti-NMDAR encephalitis is more prevalent among young women with tumours, especially ovarian teratomas. Para neoplastic autoimmunity and infection-induced molecular mimicry may explain the production of auto-antibodies against NMDAR (2). Around $41 \%$ of patients with antiNMDAR encephalitis do not have any detectable tumours (5). The diagnosis of anti-NMDAR encephalitis is usually clinched by detecting the antibodies in the patient's serum or cerebrospinal fluid (CSF). Patients with anti-NMDAR encephalitis respond poorly to standard psychopharmacological agents, but $75 \%$ of patients recover well with specific interventions such as immunosuppression and tumour resection (2). Unless the disease is diagnosed early and treated appropriately, the risk for mortality or severe disability will be as high as $25 \%$ (6). Such high mortality risk and availability of specific treatments emphasise the importance of recognising anti-NMDAR encephalitis during busy clinical practice of psychiatry (7).

In this issue, Maat et al. (8) presents the first Dutch cohort of 15 patients with anti-NMDAR encephalitis. Eighty per cent of their patients presented prominent psychiatric symptoms. Two-thirds of them were initially managed by psychiatric services and there were two fatalities. Their retrospective data corroborate prior evidence that early diagnosis of anti-NMDAR encephalitis leads to favourable clinical outcomes. They confirm that the patients with antiNMDAR encephalitis do not respond well to antipsychotic medications and benzodiazepines, but they improve considerably with immunosuppression. Their findings provide the impetus to briefly highlight the following issues, which are relevant to many clinical and academic psychiatrists.

\section{Clinical implications}

As early diagnosis and prompt management of antiNMDAR encephalitis can save many lives and can prevent severe disability, the clinical psychiatrists need to consider the following:

1. Screening all patients with first episode psychosis for anti-NMDAR encephalitis is desirable (9) but not feasible in all clinical settings. Cost-effectiveness of such screening strategy has not been evaluated so far. Hence, there is a clinical need to identify the patients at high risk by their clinical presentations. Abrupt onset, rapid progression, female gender, seizures, abnormal movements, disorientation, stupor, incontinence, fluctuations in psychopathology, autonomic dysfunction and absence of past or family history of psychiatric morbidity or substance use may indicate the investigations for anti-NMDAR encephalitis. Clinical teams should formulate local policies and explicit guidelines to facilitate early diagnosis of antiNMDAR encephalitis. 
2. Patients, who have first episode psychosis and atypical clinical presentations, often undergo neuroimaging. However, magnetic resonance imaging of the brain can be absolutely normal in $50 \%$ of patients with anti-NMDAR encephalitis (2). Electroencephalography is more sensitive, but less specific to diagnose anti-NMDAR encephalitis. Immunoassays to detect auto-antibodies should be first performed in the patients' sera. A seronegative result but high clinical suspicion of anti-NMDAR encephalitis will indicate lumbar puncture. CSF immunoassays are more sensitive than the serum assays to diagnose anti-NMDAR encephalitis (2). Once the diagnosis is made, a comprehensive evaluation for tumours is mandatory.

3. Neuropsychiatric diseases with predominant psychiatric presentations can create conflicts among the treating teams and ward managers. Clinical psychiatrists should establish multidisciplinary collaborative care including neurologists, intensivists and surgeons at the earliest to facilitate the recovery of patients with anti-NMDAR encephalitis.

4. Anti-NMDAR encephalitis may lead to longterm cognitive deficits (10). After the recovery from acute phase, clinical psychiatrists need to plan for neuropsychological assessment and cognitive rehabilitation. Despite prompt treatment, $25 \%$ of patients with anti-NMDAR encephalitis may continue to suffer moderate to severe neuropsychiatric difficulties (2). As antiNMDAR encephalitis severely disrupts one's personal, social and professional life, there is a clinical need for holistic care, psychosocial support and occupational therapy.

5. Relapses can occur in $25 \%$ of patients, especially those who do not have any removable tumour. Hence, recovered patients will require comprehensive education and longterm follow-up. A diagnosis of anti-NMDAR encephalitis does not rule out the possibility of primary psychiatric disorders later in life. Comprehensive clinical assessment, including detailed mental status examination, should consider all relevant differential diagnoses during each clinical presentation.

\section{Research directives}

Anti-NMDAR encephalitis sets the stage for investigating the NMDA glutamate receptor hypothesis for schizophrenia (11) and opens the following research avenues:

1. Clinical presentations indicating the diagnosis of anti-NMDAR encephalitis have been recognised from clinical experience and from published case series so far. There is a need for studies with appropriate control groups to identify the clinical predictors, which can distinguish those, who are likely to have anti-NMDAR encephalitis, among all patients with first episode psychosis.

2. Schizophrenia is a group of heterogeneous disorders and its aetiology is multifactorial. Between the two sets of schizophrenia and antiNMDAR encephalitis, there can be a common subset of patients with schizophrenia with NMDAR auto-antibodies, contributing to their psychopathology (3). We need better epidemiological studies to establish the prevalence of NMDAR auto-antibodies and the risk conferred by such antibodies in schizophrenia.

3. Evidence base for specific interventions to treat anti-NMDAR encephalitis is not strong. The risks and benefits of chronic immunosuppression remain uncertain (2). We need prospective intervention trials to develop standard treatment algorithms to manage anti-NMDAR encephalitis.

4. Health economic studies, evaluating the costeffectiveness of screening all patients with first episode psychosis for anti-NMDAR encephalitis and for other antibody-mediated encephalitis, are desired.

5. Immunopathogenesis of anti-NMDAR encephalitis has not been fully understood yet (2). Genetic studies, rodent models using immunisation strategies and further molecular studies may enhance our understanding of this intriguing neuropsychiatric disease.

\section{Disclosure}

The author does not have any competing interests to declare.

$$
\begin{array}{r}
\text { Rajkumar, A.P. }{ }^{1,2,3} \\
\text { M.D., D.N.B., M.R.C.Psych. } \\
{ }^{1} \text { Department of Biomedicine, } \\
\text { Aarhus University, Aarhus, Denmark } \\
{ }^{2} \text { Centre for Psychiatric research, Aarhus University } \\
\text { hospital, Risskov, Denmark } \\
{ }^{3} \text { Department of Psychiatry, Christian Medical } \\
\text { College, Vellore, India } \\
\text { E-mail: apr@hum-gen.au.dk }
\end{array}
$$

\section{References}

1. Dalmau J, Tuzun E, Wu HY et al. Paraneoplastic anti- $N$ methyl-D-aspartate receptor encephalitis associated with ovarian teratoma. Ann Neurol 2007;61:25-36.

2. Peery HE, Day GS, Dunn $S$ et al. Anti-NMDA receptor encephalitis. The disorder, the diagnosis and the immunobiology. Autoimmun Rev 2012;11:863-872. 
3. VAN DE Riet EH, Schieveld JN. First-onset psychosis, anti-NMDAR encephalitis, schizophrenia and ConsultationLiaison psychiatry. Gen Hosp Psychiatry 2013; doi: 10.1016/j.genhosppsych.2013.1001.1014. Epub ahead of print.

4. Zandi MS, Irani SR, LANG B et al. Disease-relevant autoantibodies in first episode schizophrenia. J Neurol 2011;258:686-688.

5. Dalmau J, Lancaster E, Martinez-Hernandez E et al. Clinical experience and laboratory investigations in patients with anti-NMDAR encephalitis. Lancet Neurol 2011;10:63-74.

6. Dalmau J, Gleichman AJ, Hughes EG et al. AntiNMDA-receptor encephalitis: case series and analysis of the effects of antibodies. Lancet Neurol 2008;7: 1091-1098.
7. Barry H, Hardiman O, Healy DG et al. Anti-NMDA receptor encephalitis: an important differential diagnosis in psychosis. Br J Psychiatry 2011;199:508-509.

8. Maat P, De Graaff E, Van Beveren NM et al. Psychiatric phenomena as initial manifestation of encephalitis by anti-NMDAR antibodies. Acta Neuropsychiatr 2013; doi: 10.1111/acn.12013.

9. Lennox BR, Coles AJ, Vincent A. Antibody-mediated encephalitis: a treatable cause of schizophrenia. $\mathrm{Br} \mathrm{J}$ Psychiatry 2012;200:92-94.

10. Chapman MR, Vause HE. Anti-NMDA receptor encephalitis: diagnosis, psychiatric presentation, and treatment. Am J Psychiatry 2011;168:245-251.

11. Schwartz TL, Sachdeva S, Stahl SM. Genetic data supporting the NMDA glutamate receptor hypothesis for schizophrenia. Curr Pharm Des 2012;18:1580-1592. 